\title{
Iron-deficiency Anemia Caused by a Proton Pump Inhibitor
}

\author{
Rintaro Hashimoto, Tomoki Matsuda and Akimichi Chonan
}

\begin{abstract}
A 59-year-old man was orally administered rabeprazole, a proton pump inhibitor (PPI), for gastroesophageal reflux disease, after which he gradually developed iron-deficiency anemia. The anemia did not improve following the administration of ferrous fumarate, and endoscopic screening of the entire gastrointestinal tract, including the small intestine, did not reveal any findings indicating the cause of the anemia. The patient was then switched from rabeprazole to famotidine and the anemia was cured within three months. There is much debate as to whether the long-term use of PPIs causes iron-deficiency. However, this case strongly suggests that PPIs can induce iron-deficiency anemia.
\end{abstract}

Key words: iron-deficiency anemia, proton pump inhibitor

(Intern Med 53: 2297-2299, 2014)

(DOI: 10.2169/internalmedicine.53.2743)

\section{Introduction}

Proton pump inhibitors (PPIs) are widely used and although there have been a number of reports of the risks of their long-term use, these drug are considered to be relatively safe (1). Only three cases of iron-deficiency anemia caused by PPI use have been reported $(2,3)$, and evidence suggesting a correlation is inconclusive (1). The present patient exhibited gradual iron-deficiency anemia due to the use of PPIs, and his anemia quickly improved upon PPI discontinuation. The previously reported three patients did not receive assessments of the small intestine; therefore, the possibility of small intestinal hemorrhage in these cases cannot be ruled out. However, as the present patient underwent an examination of the entire gastrointestinal tract including the small intestine, we believe that this case demonstrates that PPIs can induce iron-deficiency anemia.

\section{Case Report}

The patient, a 59-year-old man, was referred to our hospital for a detailed examination due to iron-deficiency anemia. He had a history of angina, hypertension, type 2 diabetes, dyslipidemia, reflux esophagitis, atrophic gastritis and Helicobacter pylori infection.

He had undergone Helicobacter pylori disinfection five years earlier, with bacterial elimination confirmed via the urea breath test at that time. The patient was being treated with rabeprazole, bayaspirin, ticlopidine, pitavastatin, ferrous fumarate, vitamin C, amlodipine and sodium azulene sulfonate. Rabeprazole had been prescribed for reflux symptoms in January 2011, after which the patient gradually developed anemia and was prescribed ferrous sulfate and vitamin C in September 2011. However the anemia did not improve, and he was referred to our hospital in March 2013.

A physical examination revealed moderate anemia in the palpebral conjunctiva and spoon nails. The laboratory findings are shown in Table. The data suggested typical irondeficiency anemia. The results of an occult blood test of the stool were negative.

Esophagogastroduodenoscopy showed severe atrophy (OIII) and intestinal metaplasia; however, no ulcerations, erosions or hemorrhagic lesions were observed. In addition, total colonoscopy did not show any hemorrhagic lesions, and there were no extra-ordinal lesions on contrast-enhanced CT of the abdomen and pelvis.

We therefore performed small intestine capsule endoscopy and double-balloon enteroscopy; however, no abnormal findings were detected. We considered the possibility that rabeprazole was the cause of patient's iron-deficiency anemia and discontinued the drug in June 2013 before switching to famotidine. The iron-deficiency anemia subsequently improved in September 2013 (Table), and there has since been 
Table. Hematological Findings before and after Rabeprazole Treatment

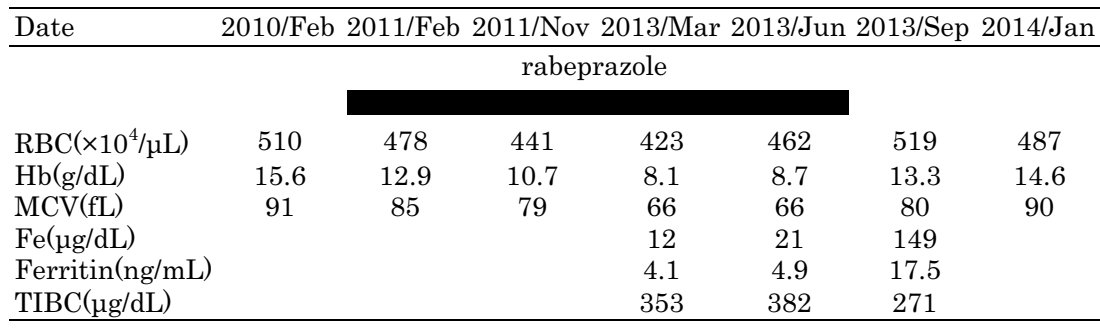

RBC: red blood cell, $\mathrm{Hb}$ : hemoglobin, MCV: mean corpuscular volume, TIBC: total iron binding capacity

no recurrence of anemia.

\section{Discussion}

The reported effects of long-term PPI use include aspiration pneumonia, Clostridium difficile infection and an increased risk of bone fractures (1). Furthermore, as gastric acid plays an important role in iron and vitamin $\mathrm{B}_{2}$ absorption, the long-term use of PPIs or $\mathrm{H} 2$ receptor agonists (H2RAs), which suppress the production of gastric acid, has been shown to increase the risk of vitamin $B_{12}$ deficiency (4).

The rate of absorption of non-heme iron is extremely poor without gastric acid, and it has long been known that iron absorption is poor under conditions of gastric acid deficiency, such as that observed in patients with atrophic gastritis (5). The risk of iron deficiency is also high after gastrectomy, and a correlation has been demonstrated between the extent of stomach resection and the serum ferritin level (6).

The effect of acid suppression by medications was initially studied using radiolabeled iron absorption tests in human volunteers, which showed a $52 \%$ decrease in absorption using antacids and $28 \%$ with low-dose cimetidine (7). While it seems possible that chronic PPI use may reduce dietary iron absorption and lead to iron-deficiency anemia, only a few studies have examined this potential association, with conflicting results.

In a study of 34 patients 6-48 months after the oral administration of omeprazole for ulcers, no decreases in the serum iron or ferritin were noted (8). However, that study comprised a small subject sample, and the serum iron and ferritin levels were not measured prior to oral administration. Another report also noted no decreases in the incidence of iron-deficiency anemia or stored iron in patients with Zollinger-Ellison syndrome (ZES) who had undergone longterm PPI treatment (9). Patients with ZES suffer from hyperacidity, and, because PPIs are unlikely to cause hypoacidity in the stomach, this report does not rule out a correlation between the use of PPIs and iron-deficiency.

A number of recent reports have stated that PPIs influence iron absorption. One report describes that PPI use increased the length of intervals between bloodletting in patients with hemochromatosis (10). Sarzynski et al. also ret- rospectively studied 98 patients who had started using PPIs and found that the hemoglobin $(\mathrm{Hb})$ level had decreased in many of the patients one year later (11). A subgroup analysis was performed in that study, and possible correlations were examined between a decreased $\mathrm{Hb}$ level and various patient attributes, such as Helicobacter pylori infection, oral antiplatelet drug use and past medical history; however, no items correlating with a decreased $\mathrm{Hb}$ level were found.

To date, there have been three reported cases of irondeficiency anemia believed to be caused by PPI use $(2,3)$. Each patient was receiving omeprazole and discontinuation of this drug led to an improvement in iron refractory irondeficiency anemia. Upper and lower endoscopy revealed no abnormal findings and while Helicobacter pylori is known to be a possible cause of iron-deficiency anemia, two of the three patients tested negative for Helicobacter infection, while the remaining patient was not tested. To the best of our knowledge, the present case is the fourth case of irondeficiency anemia believed to be caused by PPIs and the first to include an examination of the small intestine.

In the present case, iron was administered after the patient received the iron-deficiency anemia diagnosis; however, his iron-deficiency did not improve. The iron agent was nonheme iron, which requires gastric acid for absorption. The effect of iron agents in patients using PPIs is in fact poor (12), and the rate of biological iron absorption is worse with the administration of ferrous fumarate than ferrous sulfate (13). Therefore, the administration of ferrous fumarate may have been a cause of the patient's unresponsiveness to treatment in the present case.

Iron metabolism in vivo is controlled by the hepcidinferroportin axis (14). Ferroportin is an iron efflux protein and regulation of the expression level controls the absorption of iron by the body and iron reuse. Hepcidin is a liverderived protein involved in the expression of ferroportin. When hepcidin production is enhanced, such as in patients with inflammation and chronic kidney disease, iron absorption decreases (14). On the contrary, when hepcidin production is suppressed, such as patients with liver damage, the iron absorption rate rises inappropriately (15). Hence, even when iron absorption is inhibited by PPIs, the hepcidinferroportin system provides negative feedback, thereby enhancing iron absorption. Therefore, patients with irondeficiency anemia caused by PPI use may have a problem 
somewhere in the hepcidin-ferroportin system.

PPIs have greatly improved the prognosis of gastrointestinal disorders; however, the risks of the long-term use of these drugs have recently attracted attention. As PPIs are strongly suspected to have caused the iron-deficiency anemia observed in the present case, data for more cases of this nature must be compiled in the future.

The authors state that they have no Conflict of Interest (COI).

\section{References}

1. Wilhelm SM, Rjater RG, Kale-Pradhan PB. Perils and pitfalls of long-term effects of proton pump inhibitors. Expert Rev Clin Pharmacol 6: 443-451, 2013.

2. Khatib MA, Rahim O, Kania R, Molloy P. Iron deficiency anemia: induced by long-term ingestion of omeprazole. Dig Dis Sci 47: 2596-2597, 2002.

3. Sharma VR, Brannon MA, Carloss EA. Effect of omeprazole on oral iron replacement in patients with iron deficiency anemia. South Med J 97: 887-889, 2004.

4. Lam JR, Schneider JL, Zhao W, Corley DA. Proton pump inhibitor and histamine 2 receptor antagonist use and vitamin B12 deficiency. JAMA 310: 2435-2442, 2013.

5. Schade SG, Cohen RJ, Conrad ME. Effect of hydrochloric acid on iron absorption. N Engl J Med 279: 672-674, 1968.

6. Lee JH, Hyung WJ, Kim HI, et al. Method of reconstruction gov- erns iron metabolism after gastrectomy for patients with gastric cancer. Ann Surg 258: 964-969, 2013.

7. Skikne BS, Lynch SR, Cook JD. Role of gastric acid in food iron absorption. Gastroenterology 82: 1068-1071, 1981.

8. Koop H, Bachem MG. Serum iron, ferritin, and vitamin B12 during prolonged omeprazole therapy. J Clin Gastroenterol 14: 288292, 1992.

9. Stewart CA, Termanini B, Sutliff VE, et al. Iron absorption in patients with Zollinger-Ellison syndrome treated with long-term gastric acid antisecretory therapy. Aliment Pharmacol Ther 12: 83-98, 1998.

10. Hutchinson C, Geissler CA, Powell JJ, Bomford A. Proton pump inhibitors suppress absorption of dietary non-haem iron in hereditary haemochromatosis. Gut 56: 1291-1295, 2007.

11. Sarzynski E, Puttarajappa C, Xie Y, Grover M, Laird-Fick H. Association between proton pump inhibitor use and anemia: a retrospective cohort study. Dig Dis Sci 56: 2349-2353, 2011.

12. Ajmera AV, Shastri GS, Gajera MJ, Judge TA. Suboptimal response to ferrous sulfate in iron-deficient patients taking omeprazole. Am J Ther 19: 185-189, 2012.

13. López de Romaña D, Pizarro F, Diazgranados D, Barba A, Olivares $\mathrm{M}$, Brunser O. Effect of Helicobacter pylori infection on iron absorption in asymptomatic adults consuming wheat flour fortified with iron and zinc. Biol Trace Elem Res 144: 1318-1326, 2011.

14. Ganz T, Nemeth E. Hepcidin and disorders of iron metabolism. Annu Rev Med 62: 347-360, 2011.

15. Tan TC, Crawford DH, Franklin ME, et al. The serum hepcidin: ferritin ratio is a potential biomarker for cirrhosis. Liver Int 32: 1391-1399, 2012.

(C) 2014 The Japanese Society of Internal Medicine http://www.naika.or.jp/imonline/index.html 